\title{
Health 2.0
}

Mittlerweile hat das Modewort "2.0" auch den Gesundheitssektor erreicht. Es gibt Health 2.0 Konferenzen, die sich mit Themen wie Patientendaten in der Cloud oder Gesundheits-Apps auf dem iPhone beschäftigen. Die Rede ist vom 2.0 Patienten, der sich im Netz informiert, in Portalen bewertet und in sozialen Netzwerken kommuniziert. Und es gibt den Arzt 2.0, der nicht mehr nur schlicht Patienten in seiner Praxis berät und behandelt, sondern darüber hinaus auch die vielfältigen Möglichkeiten von Gesundheitstelematik und Online-Kommunikation nutzt.

Wie so oft werden auch unter Health 2.0 Datenschutz und Datensicherheit oftmals als lästiger Hemmschuh wahrgenommen, weil sie einer ungehinderten Nutzung der informationstechnischen Möglichkeiten im Wege stehen - egal, ob es um elektronische Patientenakten, Krankenhausinformationssysteme oder Cloud Computing geht. In den Beiträgen dieses Schwerpunkthefts soll es darum gehen, wie sich das Spannungsverhältnis zwischen Datenschutz und Datensicherheit einerseits und den Möglichkeiten moderner IT andererseits auflösen lässt. Hennig und Etgeton zeigen am Beispiel der "Weissen Liste" auf, dass sich bei Online-Bewertungsportalen die verschiedenen Interessen von Patient 2.0 und Arzt 2.0 (Datenschutz, Persönlichkeitsschutz, Transparenz) durchaus unter einen Hut bringen lassen. Götz nimmt die Ausgabe der elektronischen Gesundheitskarten zum Anlass, ganz grundsätzlich auf die Herausforderungen des Aufbaus einer vollständig neuen Telematik-Infrastruktur im Gesundheitswesen einzugehen. Und Menzel beleuchtet in seinem Beitrag für den Krankenhausbereich, wo der Datenschutz elektronischen Informations- und Kommunikationssystemen Grenzen setzen muss.

Eine ganz andere Ausprägung des Konflikts zwischen informationeller Selbstbestimmung einerseits und Informationsinteressen Dritter andererseits beleuchtet Damm in seinem Beitrag zu prädiktiven Gesundheitsinformationen in der modernen Medizin. In den beiden anschließenden Beiträgen geht es sodann um rechtliche und praktische Fragestellungen des Outsourcings von Datenverarbeitungsvorgängen. Biewald präsentiert mögliche Lösungsansätze, wie sich Auftragsdatenverarbeitung und ärztliche Schweigepflicht vereinbaren lassen. Herkenhöner, Fischer und de Meer zeigen an einem praktischen Beispiel auf, wie sich externe IT-Dienstleistungen im Pflegedienst verwirklichen lassen. Hase schließlich wendet sich in seinem Beitrag dem Konflikt zwischen Datenschutz- und Forschungsinteressen zu und stellt die aktuellen Regelungsansätze vor, die für mehr Datentransparenz im GKV-Bereich sorgen sollen.

Neben Health 2.0 werden in diesem Heft dann auch noch die beiden Themen polizeiliche Videoüberwachung sowie Datenschutz bei Lernsoftware behandelt. Schnabel geht in seinem Beitrag auf ein aktuelles Urteil des VG Hannover ein und erläutert in diesem Zusammenhang einige zentrale Aspekte der polizeilichen Videoüberwachung. Mester und Seifert erinnern in ihrem Beitrag daran, dass ungeachtet all der attraktiven Möglichkeiten eines Einsatzes von Lernsoftware an Schulen auch dort der Datenschutz nicht in Vergessenheit geraten darf.

\section{Benedikt Buchner}

